\title{
GÉNERO Y CULTURA GLOBAL EN EL VIDEOJUEGO ESPAÑOL
}

\author{
Lluís AnYó ${ }^{1}$ \\ Universitat Ramon Llull (Barcelona)
}

\section{A vueltas con el género videojuego}

Los videojuegos son la primera industria cultural multimedia. Ocupan un lugar central tanto desde el punto de vista económico como social. En un sentido histórico, son la primera producción cultural de masas nacida directamente de las tecnologías informáticas $y$, en un sentido industrial, su volumen de negocio es comparable al de otras grandes industrias culturales mundiales. También en España el sector de los videojuegos es uno de los que con mayor fortuna está superando los efectos de la crisis económica y financiera global:

El contexto económico actual ha provocado caídas en los ingresos de todos los sectores del entretenimiento a nivel europeo y global. A pesar de ello, la industria española del videojuego continuó siendo en 2011 la principal industria de ocio en nuestro país por volumen de facturación y sitúa a España como quinta potencia europea en consumo. A nivel europeo, el consumo global de software mantiene al videojuego como el principal segmento de ocio, superando las cifras de la música y el cine. (ADESE 2012)

Sin embargo, esta situación, aparentemente ventajosa, esconde no pocas sombras, tanto desde el punto de vista industrial como cultural. Abordaremos es-

\footnotetext{
${ }^{1}$ Agradezco a Marc Previ, de la Universitat de Barcelona, sus comentarios al texto original.
} 
tos claroscuros a través del concepto de género, que no puede definirse sin hacer referencia tanto a consideraciones de tipo formal, es decir, con respecto al texto en sí, como a otras relacionadas con su producción y consumo. Esta perspectiva nos va a permitir situar el videojuego español en el contexto mundial y comprender de este modo por qué carece, en general, de características locales. Centraremos el análisis en cinco títulos ya clásicos del videojuego de producción hispana: La pulga, La abadía del crimen, PC-Fútbol, Commandos: Behind the Enemy Lines y Castlevania: Lord of Shadows.

Consideraremos los videojuegos como artefactos culturales, en el mismo sentido que consideramos las novelas a partir del momento en que están impresas. En palabras de E. Aarseth, "computer games are games in a new material technology, just as print novels were literature in a new technology 500 years ago. Yet, it seems, we only discovered games as cultural artifacts a few years ago" (2004: 46).

Teniendo en cuenta las dificultades de definición y clasificación con las que podemos encontrarnos, parece necesario poner en cuestión el ámbito mismo de lo que entendemos por "videojuego" o por "juego de ordenador". En este sentido, Aarseth plantea la conveniencia analítica y metodológica de definir el objeto de estudio en términos de entornos virtuales, donde la experiencia del jugador, en relación directa con la jugabilidad, es decir, con el aprendizaje del juego por parte del jugador, se sitúe en el centro analítico:

Un nombre adecuado sería el de "juegos en entornos virtuales". Esta etiqueta sirve para juegos que van del Tetris pasando por Drug Wars hasta EverQuest, mientras que juguetes computerizados como Furby y juegos de dados y cartas como el Blackjack quedan excluidos. Juegos de simulación no informáticos como el Monopoly o Dungeons and Dragons no quedarían excluidos, pero quizás esto sea una ventaja más que un problema. Al fin y al cabo, la similitud entre estos y muchos juegos informatizados en entornos virtuales es innegable, así que tiene sentido incluirlos. (Aarseth 2007: 6-7)

Por supuesto, estamos de acuerdo con Aarseth en que "juegos en entornos virtuales" es un nombre muy apropiado... que la literatura especializada no utiliza. Desde nuestro punto de vista hay que rendirse a la fuerza del término videojuegos, de manera que la mejor solución sea, por un lado, definir los videojuegos en general y, por el otro, establecer una clasificación de géneros que permita diferenciar sus principales tipos.

Según Wolf y Perron (2003: 14-16), cuatro términos definen los videojuegos: algoritmo, actividad de jugar, interfaz y gráficos. Los gráficos son el aspecto más fácil de definir, puesto que se refieren a las imágenes visuales de la pantalla. En este apartado debemos incluir, aunque Wolf y Perron no lo hagan, un aspecto lamentablemente a menudo olvidado en los videojuegos -y dicho sea de paso, también en el cine-: los sonidos y la música, que contribuyen, junto con los aspectos visuales, a completar la representación en los videojuegos. La interfaz no debe confundirse con los gráficos -y el sonido-, puesto que se trata del sistema de entrada y 
salida de datos que permite comunicar al usuario con la máquina: es la pantalla interactiva que hace las veces de traductor entre el lenguaje natural del usuario y el lenguaje digital del ordenador. La actividad de jugar, quizás la más característica de un videojuego, consiste tanto en una actividad mental como física, es ergódica -término acuñado por Aarseth-y está estrechamente vinculada a la interfaz, que hace de puente entre los gráficos y los algoritmos. Además, la podemos dividir en dos áreas: la intradiegética, que es la acción de un personaje en el mundo del juego, resultado de la acción de jugar, y la extradiegética, que es la acción física del jugador para controlar el juego. Respecto al algoritmo, este es responsable de cuatro aspectos del juego: representaciones (gráficas y sonoras, incluida toda la simulación de un mundo virtual), respuestas del sistema a las acciones del jugador, reglas que regulan lo que se puede y no se puede hacer en el juego, e incluso el azar (aspectos impredecibles que algunos juegos incorporan como parte de su programación).

En este mismo sentido, una definición de los videojuegos excelente por sintética la encontramos en Juul: los videojuegos son medio-reales [half-real], están a medio camino entre las reglas del juego -mundo real- y el mundo de ficción. En un ejemplo del mismo Juul, Legend of Zelda: The Wind Waker (Nintendo, 2002), encontramos varios gráficos expresivos que muestran escenarios por donde el personaje-jugador debe viajar para rescatar a su hermana pequeña, secuestrada. Junto a estos gráficos de carácter realista y razonablemente mimético, encontramos también una flecha sobre la hermana, que nos indica a nosotros, los jugadores, que tenemos que interactuar con ella, es decir, esta flecha hace explícitas a través de la interfaz las reglas del juego que debemos seguir y que son externas al mundo de ficción en el que hay que alcanzar una serie de objetivos:

I have called video games half-real, but an alternative term would be half-fictional. On a formal level, games are themable, meaning that a set of rules can be assigned a new fictional world without modifying the rules. A game can be changed from one setting to another; the gun can become a green rectangle; the players can control wooden figures rather than humanoid characters. $\mathrm{Ne}$ vertheless, fiction matters in games and it is important to remember the duality of the formal and the experiential perspectives on fiction in games. (Juul 2005: 199)

\section{GÉNEROS: DEL CINE A LOS VIDEOJUEGOS}

Ya hemos comentado que no es posible hablar de videojuegos como de un todo homogéneo. No son lo mismo Pong (Atari, 1972), primer éxito de masas del videojuego, a principios de los años setenta, que Pokémon (Creatures, 1996) de la Gameboy, consola de Nintendo, verdadero éxito del videojuego portátil en los años noventa, o Doom (Id software, 1993), éxito shooter donde la acción es subjetiva -además de muy violenta- o Resident Evil (Capcom, 1996), característico Survival Horror y "experiencia cinemática interactiva", como bien dice F. de Felipe citando a los productores (2000: 505). 
Además, el uso que se hace de un videojuego concreto y la clasificación social que recibe dependen del lugar que ocupa en la práctica cultural más que de sus características técnicas o incluso de su forma narrativa. Al respecto, puede aclarar esta cuestión el comentario que hace J. C. Herz (1997), historiador de los videojuegos. Para él, desde el punto de vista social, los juegos electrónicos portátiles y las consolas de televisión son cosas totalmente diferentes. Los juegos portátiles eran de colores diversos y divertidos, de pequeño tamaño, funcionaban a pilas, estaban apilados en la caja de juguetes, en la habitación del niño y no en el centro del entretenimiento familiar, con el equipo musical estéreo y el todopoderoso televisor. Los juegos portátiles, de hecho, no eran más que un juguete.

Los criterios más habituales para establecer una tipología de videojuegos son el contenido o tema, la construcción del personaje-jugador y el tipo narrativo con relación a la participación del espectador-jugador. Debemos decir, sin embargo, que ninguna tipología será perfecta, es decir, habrá juegos que puedan presentar características de más de una de las categorías.

Una tipología basada en el contenido será la que más se vincule al concepto de género. En los estudios fílmicos, R. Altman define género en toda su complejidad:

La industria cinematográfica, respondiendo a los deseos del público, inicia una serie de géneros bien delimitados cuya perdurabilidad responde a la capacidad de satisfacer necesidades humanas básicas. Aunque cambien la forma predecible durante el transcurso de su vida, los géneros mantienen una identidad fundamental a lo largo del tiempo y a lo largo de la cadena que los lleva de la producción a la exhibición y el consumo por parte del espectador. La aplicabilidad de los conceptos genéricos queda garantizada por la gran variedad de significados que se atribuyen al término género así como por la función de conducto que caracteriza a la estructura textual. Desde el punto de vista privilegiado del crítico a distancia, los géneros parecen funcionar a veces como ritual y en otros momentos como ideología. (Altman 2000: 53)

El género en cine no puede entenderse como una construcción que proviene únicamente de la industria, sino que depende al mismo tiempo de la codificación de los autores del texto y de la descodificación compartida por la comunidad de gran tamaño que es el público, de modo que no es posible reducirlos a una construcción ideológica procedente de altas instancias ni tampoco a experiencias rituales elaboradas por el público. Altman define el género como una doble autoría, no adecuado para ser analizado por un modelo de comunicación frontal o lineal. En resumen, es posible que la industria cinematográfica ( $y$, debemos añadir nosotros, la industria del videojuego) se beneficie de las formas genéricas, pero no llegará nunca a controlarlas porque tendrá que compartir la autoría del género con un grupo heterogéneo, cambiante y esquivo de comunidades de espectadores.

De esta manera, debemos tener presente tanto la discursividad primaria de la industria en relación con el texto, como la secundaria constituida por la comunidad de consumidores: si la industria -del cine o de los videojuegos-define los 
géneros, la masa de espectadores los tiene que conocer y reconocer o, en caso contrario, el género no es más que una categoría vacía.

Los géneros de videojuegos, pues, a imagen de los géneros cinematográficos, no pueden entenderse como categorías transhistóricas ni tampoco como categorías claramente delimitadas libres de toda hibridación. Al contrario, los géneros son útiles en tanto que ponen en contacto diferentes intereses: regir la producción de acuerdo con un esquema o fórmula, dotar de estructura un texto, en tanto que relato, y de tema, en tanto que historia, facilitar las decisiones de marketing con respecto a la comercialización y finalmente permitir una interpretación anticipada y codificada por parte de los públicos y de los críticos, en relación con su contrato espectatorial (Altman 2000: 37 y ss.). En función de estos intereses, la adscripción al género estará poco delimitada y condicionada por el contexto histórico.

La complejidad de la adscripción genérica es, en los videojuegos, particularmente amplia. En primer lugar, por las múltiples relaciones que se establecen no solo entre los juegos de un corpus determinado, sino también entre otras formas de expresión, como, por ejemplo, el cine. En segundo lugar, por el hecho de que los videojuegos son narrativas interactivas $y$, por tanto, sus contenidos no pueden clasificarse únicamente en función del tema de la historia y el corpus de otros textos similares, sino que hay que tener presente en todo momento la forma en que el usuario participa de la historia, el grado de interactividad, la modalidad narrativa de esta, las convenciones de inmersión, etc. Valga como ejemplo la siguiente crítica aparecida en una revista especializada:

\begin{abstract}
Hay algo valioso en ser genérico, y Dead Space contiene cada una de las características del género de terror, relacionadas con experiencias pasadas que el jugador haya podido tener. Los pórticos aparentemente desiertos y los pasillos de color bronce de Dead Space recuerdan a Alien, y el terrible miedo que infundía esa película. Las criaturas retorcidas traen recuerdos de La Cosa de Carpenter, y de la sensación de asco de las pelis de Cronenberg. (Edge 2006: 25)
\end{abstract}

\title{
3. GÉNEROS DE LOS VIDEOJUEGOS
}

D. Levis, en el que es quizá el primer texto en castellano sobre el tema, propone una clasificación donde combina de forma implícita criterios de tipo temático con criterios de habilidad del jugador, advirtiéndonos que "Ios videojuegos pueden ser divididos en géneros, aunque es habitual la proliferación de productos híbridos en los que se mezclan ingredientes provenientes de géneros diferentes" (1997: 165). El resultado es la siguiente tipología: juegos de lucha, Beat'em Up o de combate, Shoot'em Up o de disparar, de plataforma, simuladores, deportes, estrategia, juegos de sociedad, lúdico-educativos y porno-eróticos. Las descripciones distan de ser sistemáticas, puesto que puede haber un criterio temático, estrictamente de género, bastante detallado, que permita diferenciar juegos Beat'em Up de juegos Shoot'em Up o de otros como los porno-eróticos, pero también un criterio basado en finalidades y competencias -los lúdico-educativos- $u$ otro basado en habilidades -como los de estrategia-. Por otro lado, 
algunos grupos, como los de lucha o combate, están diferenciados de forma muy detallada y minuciosa, mientras que otros, como los de estrategia, incluyen una amplia amalgama de videojuegos de forma indiferenciada. Esto puede explicarse en parte por la fecha del texto. Efectivamente, consideramos que la clasificación genérica de los videojuegos depende del contexto de producción y consumo, y puede cambiar de forma notable en los quince años que han pasado desde el texto de Levis, cuando el desarrollo de videojuegos de rol o de simulación social era mucho menor que en la actualidad y, en cambio, los juegos de lucha, de combate o de disparar eran muy numerosos y estaban bien diferenciados, tanto por la industria como por los consumidores.

Comparemos la anterior clasificación con una más reciente, en la que para empezar se nos explica el criterio utilizado y se nos advierte de las dificultades de la clasificación: "Los videojuegos se pueden clasificar como un género u otro dependiendo de su representación gráfica, el tipo de interacción entre el jugador y la máquina, la ambientación y su sistema de juego, siendo este último el criterio más habitual a tener en cuenta" (Belli y López Raventós 2008: 167). El resultado es un completo listado de diecisiete tipos. Constatamos que diversos tipos de juegos coinciden con la clasificación de Levis: Beat them up, lucha, deportes, plataformas; otros se encuentran más detallados: lo que antes era shoot'em ahora ocupa cuatro tipos y se divide según criterios diversos: dos según el punto de vista (acción en primera persona y acción en tercera persona), otro en función del tema (infiltración) y, finalmente, el cuarto que responde a un criterio de tipo histórico (arcade). También el tipo de simulación de combate y de carreras puede corresponder a la categoría anterior de simuladores; o los nuevos tipos de agilidad mental, educación y party game a la rúbrica ya mencionada de juegos de sociedad y lúdicoeducativos; por último, los juegos de estrategia de la primera clasificación que son aquí aventuras clásicas y gráficas; aparte se presentan los juegos en línea. Como novedad, tiene categoría propia el tipo de infiltración, definido como un juego basado "en el sigilo, la furtividad y la estrategia en vez de buscar la confrontación directa con los enemigos" (Belli y López Raventós 2008: 168).

En resumen, los mismos problemas de la clasificación de Levis pueden encontrarse en esta de ahora, pues mezcla los criterios temáticos, de finalidades, de habilidades y los históricos. Es útil constatar el carácter diacrónico de la clasificación en géneros, por la aparición, muy reveladora, de los juegos en línea, inexistente en los tipos de Levis por su muy escasa relevancia.

Aun reconociendo que toda clasificación de géneros en los videojuegos va a estar marcada por el contexto cultural de producción y consumo, y que no es posible ni quizás deseable establecer una de tipo ahistórico, ello no impide que resulte útil una clasificación en coherencia de criterios. En este sentido, recogiendo las aportaciones anteriores, proponemos como criterio a tener en cuenta en todos los casos la clase de participación del jugador en el juego, elemento diferenciador de los videojuegos en tanto que artefactos culturales y muy relevante en la orientación del consumo, mientras que el criterio narrativo o temático, el principal sin duda en las clasificaciones de género en el cine, por ejemplo, será aquí secundario. 
Dicha participación del jugador en el juego va a incluir, por un lado, la construcción del personaje, es decir, el rol, y, por otro, la construcción de la narración según sea la interacción entre personaje y jugador. En esta misma línea, la propuesta de M. J. P. Wolf es de gran interés, ya que empieza por explicitar sus criterios de clasificación. El iconográfico, a menudo utilizado en cine, no es suficiente en los videojuegos, puesto que ignora su característica diferencial principal: la experiencia de juego: "As narrative games row more complex and cinematic, we will be able to apply iconographic and thematic generic classifications from film more usefully, but interactivity will always be an important factor in the way we experience the games" (2005: 194). Además, en relación con la interactividad, se tendrá también en cuenta si el juego es multiusuario o single-player o en línea.

Wolf ordena su lista alfabéticamente, presentando nada menos que cuarenta y dos tipos distintos (2005: 196), absolutamente exhaustiva pero que por supuesto no se corresponde con los géneros habitualmente utilizados por la industria o el mercado. La principal utilidad de esta lista es ofrecer con gran detalle una amplia clasificación de los videojuegos junto con la descripción del principal elemento de participación en la experiencia de juego, lo que sirve como criterio general y se complementa con otros criterios temáticos o iconográficos.

Es necesario, sin embargo, realizar una serie de comentarios a la lista propuesta por Wolf, no para cerrar una clasificación ahistórica, tarea que nos parece tan difícil como inútil, sino para avanzar en el conocimiento del artefacto cultural videojuego y sus géneros hoy en día aceptados. En primer lugar, si comparamos los tipos de Wolf podemos reagruparlos en dos categorías muy amplias, de acuerdo también con la opinión pública no especializada: por un lado, estarían los tipos de videojuegos como tales, así reconocidos por la industria y los consumidores (abstractos, aventuras, plataforma), y, por otro lado, los tipos de habilidades o actividades requeridas al jugador (juegos de capturar, de atrapar, de persecución, de evasión, de coleccionar, etc.). Esta confusión no es rara y responde a la realidad del mercado en que algunos juegos, como hemos visto en las clasificaciones anteriores, aun siendo únicamente una actividad, se han ganado una categoría propia (estamos hablando de los juegos de disparos, combate o lucha). Otras características no relacionadas directamente con la habilidad del jugador también figuran como categoría independiente (laberinto, juego de rol, etc.) y debemos, de igual modo, tenerlas en cuenta. También encontramos, residualmente, tipos poco significativos o de los que podemos dudar, incluso, de que sean juegos (demos, diagnóstico, utilidad, película interactiva...).

\section{INDUSTRIA Y GÉNERO EN ESPAÑA}

El sector del videojuego fue declarado industria cultural en España en marzo de 2009 por parte de la comisión de Cultura del Congreso de los Diputados (Constenla 2009). Como industria, podemos considerarla la más importante del mercado del entretenimiento audiovisual español por volumen de negocio, ya que supera el consumo de cine, DVD de vídeo o CD de música. Como decíamos al inicio de este trabajo, según datos de la ADESE (Asociación Española de Distribui- 
dores y Editores de Software de Entretenimiento) en su Anuario de 2011, España se sitúa como quinta potencia europea en consumo. Los juegos para consola suponen el $93 \%$ del total, mientras que ordenadores personales, móviles u otros soportes se reparten el $7 \%$ restante. Pero, aunque la industria del videojuego en España es la primera industria del país en ocio audiovisual desde el punto de vista del consumo, no lo es desde el punto de vista de la producción. Efectivamente, si bien existe un importante número de empresas desarrolladoras, la casi completa ausencia de ayudas estatales -tanto en relación con las ayudas directas como con los incentivos fiscales- ${ }^{2}$, explica que en su mayoría estas sean pequeñas empresas especializadas en videojuegos casuales y para plataformas móviles y tablets, es decir, aquellos que, en comparación con el desarrollo de videojuegos para consola, requieren una menor inversión de producción y distribución.

Por tanto, podemos decir que el videojuego, a pesar del reconocimiento político, se encuentra desregulado, sin ayudas a la producción local o a la diversidad de contenidos, es decir, sin un reconocimiento de la producción minoritaria o alternativa que permita ir más allá de las grandes producciones multinacionales controladas por empresas no españolas. Ello a pesar de que -es nuestra opiniónlos videojuegos deben ser tenidos en cuenta en las políticas culturales no solo porque favorecen el desarrollo económico, sino sobre todo porque son portadores de valores intrínsecos de la cultura, como la identidad y la memoria colectiva, la diversidad cultural, la capacidad crítica, la expresividad y participación social, etc., de modo similar a otras formas culturales, como el cine o la literatura. Como apunta P. M. Moreno en un texto todavía vigente:

Un caso extremo en suma de concentración vertical hard y soft y producción y distribución, frente al cual las instituciones nacionales e internacionales reguladoras de la competencia empresarial deberían actuar por la simple evidencia de un abuso sistemático de posición dominante, pero cuyo valor cultural exigiría además una actuación anticoncentración al mismo título que la practicada tradicionalmente en sectores clásicos de las industrias culturales, como el libro, la radio, la televisión o la prensa. (Moreno 2003: 221)

La concentración empresarial en el caso de los videojuegos, también en España, se produce sobre todo por la videoconsola, que, recordemos, supone el $95 \%$ del total del consumo en nuestro país. En la actualidad, las videoconsolas existentes en el mercado, tanto de sobremesa-PlayStation 3, XBox 360, Wii-como portátiles -PSP, DS- pertenecen a tres empresas, ninguna de ellas de titularidad española: Sony Corporation, Microsoft Corporation y Nintendo Company. Estas diferentes consolas son incompatibles entre sí y, además, es necesario un acuerdo con la empresa propietaria para desarrollar un videojuego compatible con cualquiera de ellas.

\footnotetext{
${ }^{2}$ En España, la producción de videojuegos se sostiene en la financiación privada -merchandising, coproducción, preventas a distribuidores, inversión de capital, business angel, etc.-, aunque puede acogerse, por su carácter tecnológico, a convocatorias públicas para proyectos I+D, nacionales y europeas, como los Planes Nacionales I+D+i y otros.
} 
Con respecto a las empresas de desarrollo de videojuegos, existen distintos tipos según su titularidad: empresas de la misma propiedad que la consola, empresas participadas en mayor o menor porcentaje por las propietarias de las consolas o bien empresas independientes, que pueden fabricar para distintas plataformas, siempre a través de acuerdos. Debemos tener en cuenta, además, que la incompatibilidad de las consolas supone uno de los mayores argumentos de fidelización del consumidor, en la medida en que su adquisición supone una inversión doméstica importante.

En resumen, la producción de títulos, directa o indirectamente, se concentra sobre todo en dos países, Estados Unidos de América y Japón, y en las tres empresas, propietarias de las videoconsolas. Esta situación explica en gran parte el contenido genérico de los videojuegos, su carácter fuertemente globalizado, en tanto que no es posible rastrear verdaderas características locales -identitarias con respecto a la diversidad cultural o de otro tipo- incluso en el idioma, que suele ser el inglés o el japonés. Efectivamente, como hemos visto en el caso de los géneros cinematográficos, los géneros de los videojuegos dependerán en gran parte de sus condiciones de producción, que podemos resumir del siguiente modo: grandísima concentración vertical y estandarización de contenidos con explotación serializada de argumentos y personajes que responden a una cierta cultura global, como veremos a continuación.

El concepto de cultura global, que no vamos a desarrollar aquí, puede considerarse sinónimo de cultura mediática o cultura mainstream y se encuentra directamente relacionado con la cultura posmoderna o hipermoderna. La bibliografía al respecto es tan amplia como diversa. Diremos solamente que no se trata de un fenómeno fácil de definir, puesto que, si bien desde una cierta perspectiva, considera la cultura unificada y desterritorializada, desde otra, esa misma cultura global contiene fuerzas locales que se apropian de sus significados como forma de resistencia, identidad, etc. Por razones de claridad, limitaremos aquí la idea de cultura global a la primera perspectiva, de modo que la definiremos como "la tendencia a la uniformación de la sensibilidad a través del cine y de la televisión [y, por supuesto, los videojuegos]. Esto se consigue por la distribución de productos generados por un número cada vez menor de industrias culturales colocadas bajo la protección económica (y del gusto) de los patrones estadounidenses administrados por empresas globales" (Coelho 2009: 156).

En estas condiciones, la variación genérica de esos productos globales dependerá, por lo tanto, más del momento histórico, el año de producción y consumo, que de su carácter de producción local, en nuestro caso "española", que será, en todo momento, y como veremos, inexistente o a lo sumo insustancial.

\section{Cinco hitos del Videojuego español}

La pulga es el primer videojuego español, también conocido como Bugaboo (Portalo 2009). Lanzado en 1983 originalmente para la plataforma Sinclair ZX Spectrum por la empresa Indescomp, fue creado por Paco Suárez y Paco Portalo, y marca el inicio de lo que se ha dado en llamar la edad de oro del software español, como lo declaró así en su momento la revista especializada MicroHobby (1987). 
Aunque existen distintas versiones del juego, todas ellas tienen en común que se inician con una animación mostrando a Bugaboo, un viajero del espacio con aspecto vagamente parecido a una pulga. El jugador controla a este personaje cuyo objetivo es salir de un laberinto de grutas donde ha quedado atrapado a causa de un accidente. Los gráficos son característicos de los juegos de plataforma de la época, en un solvente 2D, y el desarrollo argumental, que existe, no es relevante. Este es un juego donde prima la acción, la habilidad del jugador para ir sorteando los obstáculos del terreno y al único enemigo, que nos van a poner difícil conseguir el objetivo. Se trata de un juego de acción característico de principios de los años ochenta, es decir, de plataforma. Hemos visto como esta categoría era bien definida por Levis y, según el mismo criterio, por Belli y López Raventós y por Wolf. Es comparable a juegos como Donkey Kong (Nintendo, 1981) o Super Mario Bros (Nintendo, 1985).

La abadía del crimen, del año 1987, es quizá el más importante videojuego de esta edad dorada de los videojuegos españoles, como afirma J. E. Gutiérrez en su excelente historia de la primera década del videojuego español (Gutiérrez 2012). Fue publicado para las plataformas Amstrad CPC, ZX Spectrum, MSX y PC (editado por Opera Soft). Obra de Francisco Menéndez con la colaboración en los gráficos de Juan Delcán, es una adaptación de la novela de Umberto Eco El nombre de la rosa. Se trata de una ambiciosa aventura gráfica en perspectiva isométrica que introduce al jugador en una abadía para llevar a cabo, siguiendo más o menos libremente el argumento de la novela, una investigación criminal, de la mano del personaje que el jugador controla en tercera persona, el monje franciscano Guillermo de Occam. La investigación se desarrolla durante siete días, en una dinámica propia de las aventuras gráficas, con diálogos en texto, recopilación de objetos de utilidad para la investigación (llaves, pergaminos, etc.), siguiendo en todo momento el ritmo de vida de una abadía franciscana. El espacio del monasterio nos puede parecer laberíntico, hasta que accedemos al auténtico laberinto del juego, que se encuentra en la biblioteca, donde Guillermo de Occam va a resolver, de nuestra mano, el misterio, si todo va bien.

Pertenece al género de aventuras, en una típica trama gráfica de misterio, donde lo más importante es el desarrollo del argumento, un argumento que depende de la capacidad de deducción del jugador y no de la rapidez de sus movimientos. Con unos gráficos en 3D de una altísima calidad, prima el mundo diegético y la historia, que debemos desentrañar en directa focalización interna fija, de sorpresa en sorpresa; en este sentido, se trata de un videojuego de brillante pátina cinematográfica y literaria. Según la clasificación de Levis, este juego corresponde al genérico de estrategia, pero no da cuenta de sus peculiares características. Sí, en cambio, Belli y López Raventós, que ya observan el tipo de aventura gráfica, así como Wolf, para quien se trata de un juego de aventuras, aunque, dada la amplitud de su lista, también tiene características de laberinto. Es comparable, por la importancia del desarrollo argumental, la capacidad reflexiva del jugador y la interfaz y los gráficos, a otros juegos de la época del tipo point-and-clic, como por ejemplo Maniac Mansion (1987), Indiana Jones and the Last Crusade (1989) o Monkey Island (1990), todos ellos de LucasFilms Games / 
LucasArts, que junto a Sierra Online fueron las productoras y distribuidoras más importantes del género.

La serie PC-Fútbol se inicia en 1992 por la editora Dinamic Multimedia y es mantenida por esta empresa en varias actualizaciones a lo largo de toda la década de los noventa. Se trata de un juego de simulación social donde el jugador dirige un equipo de fútbol, por lo que debe encargarse desde la economía hasta de planificar las tácticas. Con unas simulaciones de juego muy básicas en las primeras versiones, su fuerza se encuentra en la completa y actualizada base de datos de futbolistas y equipos, que el jugador debe gestionar con inteligencia.

Conviene detenerse en la diferencia entre este último videojuego, PC-Fútbol, y el anterior, La abadía del crimen, puesto que ambos pueden calificarse, en lenguaje común, como juegos "de estrategia". En PC-Fútbol, aunque prima la estrategia empresarial, la relación entre jugador y mundo de juego es muy distinta de la que hemos visto en La abadía del crimen, ya que que el jugador es un todopoderoso mánager que no puede personalizarse en ningún personaje concreto. Podemos decir que la estrategia de La abadía del crimen es intradiegética, que toma la forma de aventura vivida por un personaje de la mano del jugador, que se identifica con él, y en tiempo real, mientras que la de PC-Fútbol es una estrategia extradiegética. De ahí que, pese a compartir el carácter estratégico, la experiencia de juego por parte del jugador, elemento principal de nuestros criterios, impone que debamos diferenciarlos claramente el uno del otro. El aspecto fundamental de La abadía del crimen es la estrategia en una aventura gráfica mientras que el de PC-Fútbol es la simulación de un entorno empresarial y deportivo. Esta distinción, además, se corresponde con la de la producción, la prensa especializada y los consumidores. Wolf es el autor que mejor da cuenta de este tipo de juego, con su gestión simulada [Management Simulation] (Wolf 2005: 200), mientras que Levis o Belli y López Raventós no lo diferencian específicamente de otros donde la experiencia de juego es bien distinta, como la simulación de combate o carreras. Es comparable, en este sentido, a otras sagas similares, nacidas en los mismos años noventa, como FIFA Soccer Manager (EA Sports, Electronic Arts, 1997).

En este caso, resulta pertinente hacer una consideración más: en tanto que el universo diegético del juego se basa en la liga española de fútbol (aunque determinadas versiones del juego también abarcan ligas internacionales), el contenido sí está aquí determinado por la localidad de su producción y consumo, eminentemente española. Hay, pues, una cierta presencia de elementos de identidad y diversidad, aunque únicamente con respecto a los jugadores y equipos de la liga española, que son gestionados por el jugador.

Commandos: Behind the Enemy Lines es el videojuego español más vendido de todos los tiempos. Creado por Gonzo Suárez e Ignacio y Javier Pérez para Pyro Studios, y publicado por Eidos Interactive en 1998, supuso una revolución en la concepción de los juegos de táctica militar en tiempo real, incorporando exploración, sigilo y acción. Sus gráficos son excelentes y sitúan la acción en diversos escenarios de la Segunda Guerra Mundial, que toman como referentes principales el cine norteamericano. El juego se desarrolla en tiempo real, con vista isométrica. El jugador controla un grupo de soldados, seis en esta versión, que tienen que tra- 
bajar juntos para finalizar la misión, ya que se trata de personajes especializados cada uno de ellos en una única habilidad (conducción, camuflaje, disparo, etc.).

Detengámonos en este punto. Hasta ahora, la relación del jugador con el mundo del juego se podía resumir en dos extremos: podía tratarse de una relación intradiegética, directamente con un personaje, o bien de una relación extradiegética, omnipresente. En este caso, no se trata ni de una ni de la otra. El jugador toma el control de un grupo de personajes, en relación intradiegética pero no directamente relacionada con un solo personaje. Esta primera consideración nos da ya la pista del carácter híbrido del juego. Según se van completando las misiones, estas son cada vez más complejas. En ese sentido, es razonable considerar que la táctica militar junto con la destreza en el manejo combinado de los distintos personajes y los distintos objetos (armas, vehículos, etc.) sitúan este juego a medio camino de la acción y de la estrategia. Distinto de La pulga, donde primaba una habilidad simple, motriz, y de La abadía del crimen, donde la acción era insustancial, o de PC-Fútbol, todo estrategia de gestión, aquí nos encontramos con un videojuego de aventura en la variante de la táctica militar con elementos híbridos de los géneros de acción y de estrategia. Por este carácter híbrido es difícil que las anteriores clasificaciones genéricas puedan dar cuenta del juego, a no ser, como hacen Belli y López Raventós, proponiendo una categoría específica: juego de infiltración.

Para nosotros, en cambio, la hibridación genérica, lejos de ser un problema, anticipa una de las características más notables de los videojuegos del siglo xxı: la hibridación de géneros no obliga a pensar nuevos géneros sino, como en el cine, a reconocer esta característica como un rasgo típico de los artefactos culturales posmodernos. Refuerza esta idea el carácter fuertemente referencial que encontramos en la serie Commandos, con alusiones directas al cine bélico de Hollywood, como por ejemplo en la configuración de los soldados que deben cumplir las misiones, ya que estos recuerdan a equipos como los de Where Eagles Dare (MGM, 1968), The Dirty Dozen (MGM, 1967) o Force 10 from Navarone (Columbia Pictures, 1978).

En 2010 aparece Castlevania: Lord of Shadows, dirigido por Enric Álvarez para Mercury Steam por encargo de la japonesa Konami, propietaria de la serie Castlevania. El juego es una buena muestra, desde el punto de vista empresarial, del carácter claramente internacionalizado de la producción española actual, que trabaja no ya para el mercado internacional -cosa que ha hecho siempre-, sino en sistemas de producción y desarrollo globales, sin apenas distinción local de ningún tipo.

Castlevania: Lord of Shadows da gran importancia al mundo diegético en que va a desarrollarse la historia: una provincia medieval imaginaria y apocalíptica, donde el personaje controlado por el jugador, Gabriel Belmont, deberá superar una serie de niveles en lucha contra licántropos, goblins, vampiros y otros monstruos terroríficos. Los escenarios, ominosos, refuerzan la opresión y la amenaza constantes. La experiencia principal del jugador con respecto al juego, directamente conectado con el personaje de Gabriel, es la acción, la lucha, la superación de los obstáculos físicos. Sin embargo, y de la mano de unos gráficos 
hiperrealistas, el mundo del juego y la historia que nos cuenta, que nos contamos al jugarlo, es fundamental para su disfrute. De nuevo nos encontramos con una cierta hibridación genérica en un juego con importantes elementos de acción -incluso de beat'em up en los combates cuerpo a cuerpo- en el marco de un universo diegético muy cuidado, que establece las claves de una relación de tipo cinematográfico entre el jugador y su personaje, y también con el espacio y los otros actores de la aventura. Sin duda, se trata de un videojuego que combina con habilidad los géneros de acción y de aventura. Una hibridación que se refiere, por un lado, como podemos ver, a la propia adscripción genérica del juego, pero también a los referentes que utiliza, como en el caso de Commandos, propios de una cultura global fuertemente basada en el cine mainstream de origen norteamericano, y, en absoluto, a algún rasgo culturalmente distintivo de acuerdo con su país de producción, ni siquiera el idioma. Así, Castlevania: Lord of Shadows, parece retomar para la construcción de su universo diegético elementos de la literatura de fantasía épica, como los clásicos El señor de los anillos (1954-55) y Las crónicas de Narnia (1951-54) de J. R. R. Tolkien y C. S. Lewis, respectivamente, ambos llevados al cine en la primera década del siglo xxı en productos blockbuster masivos y de consumo global.

Castlevania constituye un muy buen ejemplo de lo que estamos comentando. Se trata de una serie iniciada en 1986 siempre bajo la propiedad de la japonesa Konami Corporation, y que sigue en activo, a la espera de la aparición del título Castlevania: Lord of Shadows 2 para 2013. Los primeros títulos, en consonancia con el año de publicación, son en general aventuras de plataforma, con gráficos en 2D y, en cuanto al elemento diegético, fantasías de terror, puesto que se refieren al castillo del Conde Drácula y la mitología cinematográfica en torno a los vampiros y otros monstruos clásicos del terror. La serie evoluciona poco a poco, en sus diferentes episodios, introduciendo por ejemplo gráficos en 3D en Castlevania 64 (1999) y Castlevania: Legacy of Darkness (1999). En esta última entrega, como hemos visto, los referentes diegéticos, aunque mantienen elementos propios del cine de terror, derivan hacia las sagas de fantasía épica. Su jugabilidad es característica de juegos de aventura-acción, como la saga God of War (SCE Santa Monica Studio, Sony Computer Entertainment, 2005-13), inspirada en la mitología griega.

Una vez más, pues, las mayores diferencias genéricas entre los distintos títulos de Castlevania se explican por su año de producción y no por su país de origen. Esta afirmación puede aplicarse a otros casos como La pulga, La abadía del crimen, PC-Futbol o Commandos. Efectivamente, además de la importancia del momento histórico para situar un videojuego en su género, cabe insistir en su carácter esencialmente marcado por una cultura global, puesto que los videojuegos que hemos analizado, aunque de producción española, se inscriben de forma clara, tanto por lo que se refiere a la jugabilidad como a la diégesis, en relación a otros juegos contemporáneos de producción anglosajona o japonesa y no hispana, e incluso encuentran sus referentes en otras formas expresivas como el cine o la literatura, que también forman parte de esa misma cultura global. 


\section{CONCLUSIÓN: HIBRIDACIÓN DE GÉNEROS Y CULTURA GLOBAL}

En estas condiciones, una definición de los géneros de los videojuegos que no tenga en cuenta aspectos locales pero sí históricos se ajustará con probabilidad a la realidad de producción y consumo tanto en España como en otros países europeos y occidentales, sin diferencias significativas entre ellos. A efectos de nuestra propuesta, el concepto de experiencia de juego como forma de participación nos servirá de guía para establecer una clasificación de género, siempre condicionada más por el año que por el lugar de producción.

Habrá que tener en cuenta que el aspecto iconográfico es el que determina con más claridad el momento histórico del juego, por ejemplo para tipos como los de plataforma. Los gráficos hay que relacionarlos con lo que podemos llamar la puesta en escena y la interfaz. En este sentido, la principal diferencia se produce entre un juego de base textual y un juego gráfico y, dentro de este último, en 2D o en 3D.

Asumiendo toda esta complejidad, podemos proponer la siguiente tipología, que toma como criterio principal la experiencia de juego del usuario. Otros aspectos como el corpus temático, el carácter narrativo, la estructura de juego o la modalidad de jugador permiten afinar la clasificación, al mismo tiempo que intentamos que no se aleje de las categorías habitualmente aceptadas por la industria y los consumidores. Se trata, además, de un conjunto de tres grandes géneros que a su vez pueden dividirse en subgéneros específicos:

-Acción: la habilidad principal consiste en tener unos buenos reflejos para combatir al enemigo. El usuario debe reaccionar con rapidez y la atención se encuentra muy focalizada. No excluyen la narración, pero esta no los define. Los podemos subdividir en juegos shooter, como Quake (Id software, 1996) o Doom (Id software, 1993), que normalmente incluyen una violencia muy primaria, narrada en primera persona, incorporan en su configuración espacial el laberinto y se presentan en modalidad de juego multiusuario en línea; juegos de arcade, como Pong (Atari, 1972) o Space Invaders (Taito, 1978), un tipo muy localizado en el tiempo; los de plataforma como Prince of Persia (Broderbund, 1989) o SuperMario Bros (Nintendo, 1985); los de combate y lucha como Tekken (Namco, 1994) o Mortal Kombat (NetherRealm Studios, 1992); y los de deporte como FIFA (EA Sports, 1993). A esta categoría pertenece La pulga.

-Aventura: en un mundo recreado, el jugador debe controlar los recursos y defenderse de los ataques de los enemigos para tener éxito. Prima la reflexión y la duración del juego por encima de las reacciones rápidas. Por su complejidad, incluyen a menudo elementos tales como un espacio laberíntico que hay que recorrer o una serie de enigmas que hay que resolver, con manipulación de objetos (tipo puzle). Presentan un claro desarrollo argumental y de los personajes (complejidad diegética) y la implicación intradiegética por parte del jugador. En este sentido, la inmersión que experimenta el jugador en el mundo de ficción es mayor que en otros tipos de juegos y, por tanto, puede ser tenida en cuenta también como un elemento de diferenciación entre los diversos géneros. En cuanto al desarrollo argumental este es fundamental, como en Police Quest (Sierra Online, 
1987) o Manic Mansion (LucasArts, 1987), auténticas ficciones interactivas. Dentro de este grupo hay diferentes subcategorías según la interfaz (textual o gráfica). También hay variaciones temáticas determinantes, ya que, al ser sustanciales los aspectos diegéticos, el tema, como en el cine, determina en gran medida el género, como por ejemplo la acción-aventura de Tomb Raider (Core design, 1996), Myst (Cyan, 1993) o GrandTheft Auto (RockStar, 2001), el Survival Horror de Alone in the Dark (Infogames, 1992) o Resident Evil (Capcom, 1996), y la estrategia militar de Medal of Honor (DreamWorks Interactive, 1999). Pertenecen a esta categoría La abadía del crimen y, en gran medida, Commandos: Behind the Enemy Lines así como Castlevania: Lord of Shadows. En esta tipología apreciamos una subcategoría con respecto a aquellos videojuegos en los que prima el personaje (aunque el desarrollo argumental sigue siendo importante), como en Diablo (Blizzard, 1996) o Final Fantasy (Square, 1987). Si es masivo y en línea, se trata de los Massively Multiplayer Online Role Playing Game (MMORPG), que, por su importancia, podemos considerar un grupo aparte, como EverQuest (Sony Online, 1999) o World of Warcraft (Blizzard, 2004).

-Simulación: el jugador actúa como el conductor de una máquina (un avión, un helicóptero, etc.) o bien como el dios de un entorno social más o menos amplio. Prima la estrategia para controlar la máquina o el entorno social, es decir, la habilidad para dirigir o asumir el control de una tecnología o un espacio colectivo, donde el jugador desempeña el papel de figura omnipresente sin una relación directa con un personaje, esto es, sin una relación claramente intradiegética. Dentro de este grupo hay dos subgrupos claramente diferenciados: la simulación tecnológica, como Flight Simulator (Psion, 1983), y la simulación social o situacional, como Age of Empires (Ensemble, 1997) o The Sims (EA, 2000). Pertence a esta categoría PC-Futbol.

-Abstractos, tradicionales, educativos: pertenecen a esta categoría independiente los juegos abstractos que a menudo son la adaptación informática de juegos tradicionales (ajedrez, damas, puzzles) o que poseen una finalidad educativa. Quizá el más conocido de este grupo sea el Tetris (Elorg, 1985).

Como hemos visto, los géneros en los videojuegos plantean problemas similares a los que podemos encontrar en el cine, acentuados por una mayor complejidad en la relación del jugador con el juego, de modo que los elementos diegéticos, que deben ser siempre tenidos en cuenta, no son lo fundamental, sino que se subordinan a la actividad misma de juego, la relación entre el jugador y el juego. Dicha relación es el criterio principal a tener en cuenta para la clasificación de los géneros en los videojuegos. De esta forma, la jugabilidad debe centrar la reflexión académica como lo hace en la producción y en el consumo y la crítica especializada.

Según este criterio, habría tres grandes géneros, aceptados en la práctica corriente del mercado, que son: acción, aventura y simulación. Un cuarto género, el de los videojuegos abstractos, resulta necesario para completar la clasificación.

Los videojuegos españoles participan, como es natural en una industria totalmente globalizada, de estas mismas características. Además, la hibridación de los géneros es cada vez más una constante en los títulos recientes, en los que 
a la jugabilidad se une un completo universo diegético, a menudo de la mano de claros referentes cinematográficos. Aunque antes hemos clasificado en el género de aventura Commandos: Behind the Enemy Lines y Castlevania: Lord of Shadows, insistimos en que su adscripción es híbrida y participan tanto de la aventura como de la acción.

Los géneros de los videojuegos, como, de otro lado, los de otros artefactos culturales, dejan de ser puros y se hibridan en muy distintas, y, es de esperar, en muy inesperadas combinaciones.

\section{OBRAS CITADAS}

Aarseth, Espen (2004): "Genre Trouble: Narrativism and the Art of Simulation". En: Wardrip-Fruin, Noah y Harrigan, Pat (eds.): First person. New media as story, performance and game. Cambridge, The MIT Press, pp. 45-55.

- (2007): "Investigación sobre juegos: aproximaciones metodológicas al análisis de juegos". En: Artnodes, n. 7 . Disponible en <http://artnodes.uoc.edu>. Última visita: 10.02.2009.

ADESE (2012): "Anuario de la industria del videojuego2011". En: http://www.adese.es/ docs/documentacion/resultados-anuales/memoria_adese_2011.pdf. Última visita: 25.01.2013.

Altman, Rick (2000): Los géneros cinematográficos. Barcelona, Paidós.

Belli, Simone y López Raventós, Cristian (2008): "Breve historia de los videojuegos". En: Athenea, n. ${ }^{\circ}$ 14, pp. 159-179. Disponible en <http://psicologiasocial.uab.es/athenea/index.php/atheneaDigital/issue/view/16>. Última visita: 23.11.2008.

Coelho, Teixeira (2009): Diccionario crítico de política cultural. Cultura e imaginario. Barcelona, Gedisa.

Constenla, Tereixa (2009): "Un respeto para el videojuego". En: El País (Suplemento Vida \& Artes), 26 de marzo, p. 36. Disponible en <http://www.elpais.com/articulo/ cultura/respeto/videojuegoelpepucul/20090326elpepicul_2/Tes>. Última visita: 23.05.2010.

De Felipe, Fernando (2000): "A las puertas del cine interactivo: videojuegos y narrativa potencial". En: Trípodos, n. ${ }^{\circ}$ extra, pp. 497-508.

Edge (2006): "Sopa de géneros recalentada. Es necesario renovar el menú". En: Edge. Video Game Culture, n. ${ }^{\circ} 28$, p. 25.

Gutiérrez, Jaume Esteve (2012): Ocho quilates. Una historia de la edad de oro del software español. Barcelona, STAR-T Magazine Books.

Herz, J. C. (1997): Joystick Nation. How Videogames Ate our Quarters, Won our Hearts, and Rewired our Minds. Nueva York, Little brown.

Juul, Jesper (2005): Half-real. Video Games between Real Rules and Fictional Worlds. Cambridge, The MIT Press.

Levis, Diego (1997): Los videojuegos, un fenómeno de masas. Barcelona, Paidós.

Moreno, Pedro M. (2003): "El videojuego: lecciones de la primera cultura multimedia". En: Bustamante, Enrique (ed.): Hacia un nuevo sistema mundial de comunicación. Barcelona, Gedisa, pp. 207-226. 
Portalo, Francisco (2009): "Bugaboo, un hito en la historia del Software Español". En: <http://www.bugabootheflea.com/bugaboo.pdf>. Última visita: 25.01.2013.

[s. a.] (1987): "El videojuego español invade Europa". En: MicroHobby, n. ${ }^{\circ} 110$, pp. 18-21.

Wolf, Mark J. P. (2003): "Introduction". En The Video Game Theory Reader. Nueva York, Routledge,

(2005): "Genre and the Video Game". En: Raessens, Joost y Goldstein, Jeffrey (eds.): Handbook of computer game studies. Cambridge, The MIT Press, pp. 193-204. 